Cahiers d'études africaines

240|2020

Soudan : identités en tension

\title{
GASMELBARI Suhaib. - Talking about Trees
}

\section{Anne-Laure Mahé}

\section{OpenEdition \\ Journals}

\section{Édition électronique}

URL : https://journals.openedition.org/etudesafricaines/32898

DOI : 10.4000/etudesafricaines.32898

ISSN : 1777-5353

\section{Éditeur}

Éditions de l'EHESS

\section{Édition imprimée}

Date de publication : 2 décembre 2020

Pagination : 1029-1031

ISSN : 0008-0055

\section{Référence électronique}

Anne-Laure Mahé, "GasmelBaRI Suhaib. - Talking about Trees », Cahiers d'études africaines [En ligne], 240 | 2020, mis en ligne le 02 décembre 2020, consulté le 06 janvier 2023. URL : http://

journals.openedition.org/etudesafricaines/32898; DOI : https://doi.org/10.4000/etudesafricaines. 32898

Ce document a été généré automatiquement le 6 janvier 2023.

Tous droits réservés 


\title{
GASMELBARI Suhaib. - Talking about
}

\section{Trees}

\author{
Anne-Laure Mahé
}

\section{RÉFÉRENCE}

GASMELBARI Suhaib. - Talking about Trees. France, Soudan, Allemagne, Tchad, Qatar, Agat Films \& Cie, Made in Germany Filmproduktion GmbH, Goï-Goï Productions, 2019.

1 Tourné en 2015, Talking about Trees, du réalisateur soudanais Suhaib Gasmelbari, suit les péripéties des membres septuagénaires du Sudanese Film Group (SFG), Ibrahim Shaddad, Manar al Hilo, Suleiman Mohamed Ibrahim et Altayeb Mahdi, alors qu'ils tentent d'organiser la projection d'un film dans un cinéma désaffecté d'Omdurman. Faisant l'économie des dispositifs classiques du cinéma documentaire - la voix off ou les entretiens en face à face -, le réalisateur laisse les enjeux de ce film à la fois comique et tragique affleurer progressivement, faisant confiance à son spectateur pour collecter les indices et comprendre de quoi il est ici véritablement question.

Car c'est bel et bien d'arbres qu'il s'agit, comme le souligne le titre tiré d'un poème écrit par Bertolt Brecht en 1939: «Que sont donc ces temps, où / Parler des arbres est presque un crime / Puisque c'est faire silence sur tant de forfaits ! » À travers ces vers, le dramaturge allemand pose la question d'une pratique artistique devenue impossible et vide de sens si elle ne confronte pas le dramatique contexte politique dans lequel elle prend place. Or, Gasmelbari démontre que dans le Soudan d'Omar Al-Bechir - il dirigea le pays de 1989 à 2019 - parler des arbres, ici du cinéma, implique nécessairement de parler du pouvoir et de ses forfaits, en particulier parce que cette activité est justement traitée comme subversive par le régime. Mais lorsque ce dernier politise les activités les plus banales, il donne en même temps naissance à de nouveaux sites de résistances, au point que des personnes âgées rejouant la scène finale de Sunset Boulevard au cours d'une panne d'électricité puissent incarner la résistance quotidienne de James C. Scott ${ }^{1}$ ou les pratiques discrètes de contention de Johnston et Mueller ${ }^{2}$. 
Cette politisation du cinéma devient évidente lorsque se pose la question d'obtenir les autorisations officielles nécessaires à la projection. Nos héros ont trouvé le lieu - non sans ironie nommé Cinéma de la Révolution (Sinema Ath-Thawra) ${ }^{3}$ - et choisi le film - Django Unchained (2012, Q. Tarantino), sur la recommandation des jeunes du quartier - mais ils doivent maintenant faire face aux méandres kafkaïens d'un régime connu pour son utilisation stratégique des formalités administratives. La demande du SFG passera ainsi entre les mains d'au moins quatre administrations différentes, dont les services de sécurité et le ministère de la Culture et de l'Information, qui se renvoient la balle et demandent sans cesse davantage d'explications. Les réalisateurs décident finalement d'abandonner le projet. Évoquant la forme du whodunit, le film répond alors de façon définitive à la question posée dès la seconde scène, lorsque les animateurs d'une émission de radio demandent qui a tué le cinéma soudanais : c'est le régime de Bechir. Il n'a pas été la victime imprévue de l'instauration d'un couvre-feu dans un pays où la majeure partie des salles était en plein air, mais bel et bien sciemment assassiné. L'un des interlocuteurs du SFG au sein de l'administration admet d'ailleurs qu'il s'agissait d'une décision politique, suscitant l'étonnement, mais aussi la satisfaction, de Shaddad. C'est que dans un régime caractérisé par son usage récurrent de la dissimulation et du mensonge, un tel aveu constitue une petite victoire, une rupture minime mais symbolique de l'ordre établi. À un niveau plus personnel, cela représente peut-être un début de justice pour ces quatre réalisateurs, formés durant la période communiste du Soudan (1969-1983), pour certains en Allemagne de l'Est et en URSS, dont les rêves furent brisés par la révolution islamiste.

En dévoilant les logiques de fonctionnement du régime et les façons dont certains tentent d'y résister, le film parle finalement d'un arbre en particulier : celui qui se trouve sur le logo du National Congress Party, le parti hégémonique qui a dominé le pays jusqu'en avril 2019. Il montre à quel point ses racines sont profondes à travers l'une des scènes les plus drôles du film, lorsque se pose la question de la logistique de l'organisation d'une projection dans un cinéma en plein air entouré par six mosquées, donc six muezzins, donc six appels à la prière simultanés. Les quatre cinéastes notent qu'il n'y en avait pas autant auparavant, sous-entendu avant 1989. Gasmelbari touche ici à la question de l'ancrage quotidien du régime : s'il est possible de rire du pouvoir et de ses absurdités, il n'en reste pas moins que son projet révolutionnaire d'ingénierie sociale basé sur l'islamisation et l'arabisation a transformé le quotidien des Soudanais. Les appels à la prière qui se font entendre tout au long du film fonctionnent comme un rappel constant de cette réalité matérielle de la domination autoritaire, répondant à une question soulevée en 1997 par Marc Lavergne et Roland Marchal ${ }^{4}$ : l'expérience islamiste a-t-elle échoué?

Bien qu'il donne à voir avant tout comment la répression peut fonctionner par la contrainte administrative et l'autocensure, le film n'évacue pas totalement la question de l'usage de la violence physique. Elle est retranscrite dans les propos de Shaddad, lorsqu'il évoque son emprisonnement au début des années 1990, dont des éléments se retrouvent dans le court-métrage qu'il réalise et qui dépeint des hommes seuls, enfermés dans des toilettes comme il le fut, une goutte d'eau leur tombant à intervalle régulier sur le sommet du crâne. Si les lignes rouges à ne pas franchir afin d'éviter d'être confronté à cette violence ne sont jamais clairement énoncées, elles transparaissent à certains moments : lorsque Shaddad évite d'énoncer clairement que c'est le régime qui a tué le cinéma, ou encore lorsque nos héros s'interrogent sur la 
stratégie à adopter face aux autorités et la nécessité d'éviter de se faire soupçonner - de façon sincère ou instrumentale - d'être financés par l'étranger. Ces moments d'autocensure ne peuvent être compris indépendamment de la capacité du régime à exercer un fort niveau de violence en dernier recours.

6 Face à l'apparition progressive et de plus en plus évidente d'une ligne rouge, les membres du SFG abandonnent leur projet. S'agit-il cependant d'un échec? Le film se conclut par l'organisation d'une séance de cinéma dans un village, comme nous avons $\mathrm{vu}$ les quatre réalisateurs le faire auparavant. Ils ont renoncé à la projection d'Omdurman afin de pouvoir continuer à organiser ces événements plus modestes. Or, le film a bien montré que ce n'est pas tant le contenu des films qui est jugé subversif par les agents du régime, mais le cinéma en tant qu'institution et pratique : un lieu où l'on se rassemble, où l'on se rencontre, où l'on se parle. L'interprétation reste donc ouverte : l'abandon du projet relève-t-il de la soumission volontaire ou d'une stratégie de résistance?

7 Constituant un témoignage riche sur ce que vivre dans un régime autoritaire signifie au quotidien ainsi que sur les logiques de fonctionnement du régime qui domina le Soudan pendant trente ans, Talking about Trees soulève donc des questions centrales aux études sur le pouvoir autoritaire et la contestation en contexte non démocratique. L'interprétation des comportements des individus sur lesquels s'exerce le pouvoir reste un puzzle théorique et empirique majeur, certains auteurs voyant du consentement là où d'autres voient de la subversion en fonction du cadre théorique et de la méthodologie adoptée. On peut regretter que l'absence d'entretiens avec les membres du SFG ne nous permette pas d'avoir accès aux significations qu'eux-mêmes confèrent à leurs actions. Une telle conversation serait certainement plus aisée aujourd'hui, suite au renversement du régime en avril 2019. Cet événement colore inévitablement la lecture du film en lui conférant a posteriori une issue plus heureuse. La présence importante des artistes au cours de la mobilisation qui a contribué au départ de Bechir rappelle d'ailleurs que la pratique artistique n'est en effet jamais anecdotique dans de tels contextes: outil et espace de production de projets de contre-société, elle est fondamentalement politique.

\section{NOTES}

1. J. C. ScotT, Weapons of the Weak: Everyday Forms of Peasant Resistance, New Haven, Yale University Press, 1985.

2. H. J OHNSTON \& C. M Ueller, «Unobtrusive Practices of Contention In Leninist Regimes », Sociological Perspectives, 44 (3), pp. 351-375.

3. Le cinéma doit son nom au quartier dans lequel il est situé, celui d'al-Thawra à Omdurman, ville jumelle de Khartoum.

4. M. LAVERGNE \& R. MARCHAL, « Le Soudan, l'échec de l'expérience islamiste? », Politique africaine, 66, 1997. 


\section{AUTEURS}

\section{ANNE-LAURE MAHÉ}

Institut de recherche stratégique de l'École militaire, Paris 